\title{
FINITE ELEMENT SOLUTION OF DYNAMIC RESPONSE OF HELICAL SPRINGS
}

\author{
Ayadi, S. \& Hadj-Taïeb, E. \\ Unit of Applied Fluid Mechanics and Modelling, \\ National Engineering School of Sfax, BP. W, 3038 Sfax, Tunisia \\ E-Mail: samya@netcourrier.com; ezed.hadj@enis.rnu.tn
}

\begin{abstract}
A numerical solution is presented to describe wave propagations in axially impacted helical springs. The governing equations for such problem are two coupled hyperbolic, partial differential equations of second order. The axial and rotational strains and velocities are considered as principal dependent variables. Since the governing equations are non-linear, the solution of the system of equations can be obtained only by some approximate numerical simulation. The finite element method, based on the Galerkin formulation, is applied to discretize the mathematical equations leading to a non-linear system of equations solved by an iterative Gauss substitution method. The computed results describe the evolution of axial and rotational strains and velocities, in different sections of the spring and show the interaction between axial and rotational waves. To validate the reliability of the model presented herein, the numerical results are compared with those of other workers obtained by the method of characteristics.

(Received in May 2007, accepted in September 2007. This paper was with the authors 2 months for 2 revisions.)
\end{abstract}

Key Words: Helical Spring, Dynamic Response, Strain, Finite Element Method, Non-linear Behaviour

\section{INTRODUCTION}

The helical spring is the simplest element which is used in the mechanical engineering and which can be found in many apparatuses, as the balances, the brakes and the suspensions. It makes it possible to maintain a tension or a force in a mechanical system, to absorb the shocks and to reduce the vibrations. To simplify the analysis; it is generally assumed that the material is elastic. The design of helical springs requires two stages as the static and dynamic. The analytical solution of the static response of cylindrical helical springs subjected to large deflections was achieved by Love [1].

The dynamic response of helical springs can be broadly characterized as linear or non-linear. In the past, the dynamic response is treated by restricting the analysis to small displacements about an equilibrium position. Therefore, the oscillatory system can be assumed to be linear. Costello [7] presents a work on the significance of torsional oscillations on the radial expansion of helical springs. In this work a linear theory was presented and the analytical solution, obtained by the Laplace transform, did indicate rather large radial expansion under impact. Ayadi and Hadj-Taïeb [2, 3] resolved numerically the linear model of Costello by using the method of characteristics and the finite difference method of Lax-Wendroff.

When a helical spring is subjected to a rather large impact loading, significant torsional oscillations can occur in the spring and the behaviour of the spring becomes non-linear. The one dimensional equations of motion, governing this behaviour, are derived in an article by Phillips and Costello [12].

Stokes [14] conducted an analytical and experimental program to investigate the radial expansion of helical springs due to longitudinal impact. 
In many research papers (Yilderim [15-17], Becker [18], Lee and Thomson [26], Mottershead [11]) the analysis of the dynamic response of elastic material springs is investigated using dynamic stiffness and transfer matrix methods. Equations of helical spring motion were used for predicting the natural frequencies.

Sinha and Costello [13] used a finite difference technique and the method of characteristics to solve numerically the non-linear partial differential equations in the time domain. They simplify the experimental velocity curve of the impacted end of the spring obtained by Costello [7]. The obtained results illustrate the axial and rotational strains only at the impacted end of the spring. However, the finite difference method results in too many oscillations.

Finite element solutions for complicated partial differential equations arising from various problems in applied mechanics are well documented in such books as those by Zienkiewicz and Taylor [23], Desai and Abel [21], and Norrie and de Vries [20]. This method has the attractive features of flexibility, generality, and consistency.

Cook and Young [6] used a simple finite element procedure to compute the deflection and state of stress in a closely-coiled helical spring. Dammak et al. [8] and Yutaka et al. [25] applied the finite element to determine the distribution of the stresses along the spring. Forrester [9] applied the finite element method to evaluate the stiffness matrix due to a unit axial load.

The purpose of the work described herein is to present a finite element solution for the one-dimensional dynamic response equations of helical springs. The solution method is described first, and then this is followed by some examples of the comparison of numerical results with those found in the work of Sinha and Costello [13]. The strain and velocity time-history for linear and non-linear models are presented and compared in some sections of the spring.

\section{MATHEMATICAL MODELS}

The equations which describe non-linear one-dimensional dynamic behaviour of helical springs can be adapted from the analytical model developed by Phillips and Costello [12]. Applying the theory of dimensional analysis and the momentum equations, to an element of spring between two sections $x$ and $x+d x$, submitted to axial force $F$ and torque $T$ (Fig. 1a) yields the following equations of spring motion:

$$
\begin{aligned}
& a \frac{\partial^{2} u}{\partial x^{2}}+b \frac{\partial^{2} v}{\partial x^{2}}=e \frac{\partial^{2} u}{\partial t^{2}} \\
& b \frac{\partial^{2} u}{\partial x^{2}}+c \frac{\partial^{2} v}{\partial x^{2}}=e \frac{\partial^{2} v}{\partial t^{2}}
\end{aligned}
$$

where $u$ is the axial displacement of the spring, $v=r \theta$ is the rotational displacement of the spring, $r$ is the radius of the spring helix in the unstretched position, $\theta$ is the rotation of the spring, $x$ is the axial co-ordinate along the spring and $t$ is time. The coefficients $a, b, c$ and $e$, occurring in equations (1) and (2), are given by the expressions:

$$
\begin{gathered}
a=\frac{r^{2}}{E I} \frac{\partial F}{\partial \varepsilon}=\left(v_{x} \sin \alpha+\cos \alpha\right)(\sin \alpha)\left\{\frac{-v}{1+v}\left(v_{x} \sin \alpha+\cos \alpha\right)+\frac{\cos ^{2} \alpha}{\left[1-\left(1+u_{x}\right)^{2} \sin ^{2} \alpha\right]^{3 / 2}}\right\} \\
b=\frac{r^{2}}{E I} \frac{\partial F}{\partial \beta}=\frac{r}{E I} \frac{\partial T}{\partial \varepsilon}=\sin ^{2} \alpha\left\{\frac{\left(1+u_{x}\right) \cos ^{2} \alpha}{\left[1-\left(1+u_{x}\right)^{2} \sin ^{2} \alpha\right]^{1 / 2}}-\frac{\cos \alpha}{1+v}-\frac{2 v}{1+v}\left(1+u_{x}\right)\left(v_{x} \sin \alpha+\cos \alpha\right)\right\} \\
c=\frac{r}{E I} \frac{\partial T}{\partial \beta}=\sin \alpha\left[1-\frac{v}{1+v}\left(1+u_{x}\right)^{2} \sin ^{2} \alpha\right]
\end{gathered}
$$




$$
e=\frac{M r^{2}}{E I h}
$$

where $h$ is the length of the spring in the unstretched position, $E$ is the Young modulus of the spring material, $M$ is the total mass of the spring, $I$ is the moment of inertia of the wire cross section, $v$ is Poisson's ratio of the spring material and $\alpha$ is the helix angle of the spring in the unstretched position. Thus it is seen that the coefficients $a, b$ and $c$ are functions of the axial and rotational strains $\varepsilon=u_{X}=\partial u / \partial x$ and $\beta=v_{X}=\partial v / \partial x=r \partial \theta / \partial x$ and hence, the governing equations of motion are non-linear.

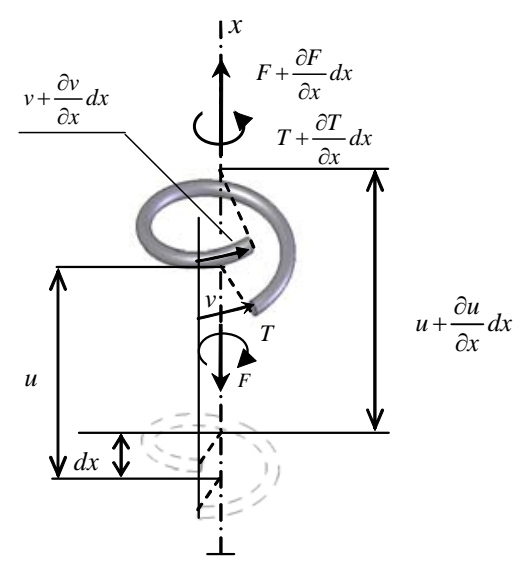

a) Free body diagram of spring element

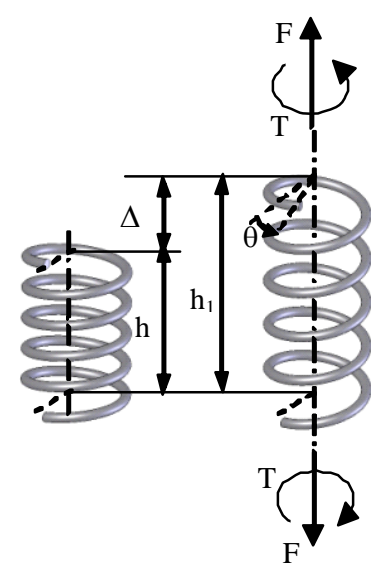

b) Static deflection of helical spring

Figure 1: Helical spring description.

It can be seen from equations (3), (4) and (5) that when the strains are small, i.e. $\left|u_{x}\right|<<1$ and $\left|v_{X}\right|<<1$, these coefficients have the approximate constant values:

$$
a=\left(1-\frac{v}{1+v} \cos ^{2} \alpha\right) \sin \alpha, b=-\frac{v}{1+v} \sin ^{2} \alpha \cos \alpha, c=\left(1-\frac{v}{1+v} \sin ^{2} \alpha\right) \sin \alpha
$$

and the equations of motion are rendered linear.

Since:

$$
\frac{\partial u_{x}}{\partial t}=\frac{\partial u_{t}}{\partial x} \text { and } \frac{\partial v_{x}}{\partial t}=\frac{\partial v_{t}}{\partial x}
$$

equations (1) and (2) can be converted into a set of first order partial differential equations:

$$
\begin{gathered}
\frac{\partial u_{t}}{\partial t}-\frac{a}{e} \frac{\partial u_{X}}{\partial x}-\frac{b}{e} \frac{\partial v_{X}}{\partial x}=0 \\
\frac{\partial u_{X}}{\partial t}-\frac{\partial u_{t}}{\partial x}=0 \\
\frac{\partial v_{t}}{\partial t}-\frac{b}{e} \frac{\partial u_{X}}{\partial x}-\frac{c}{e} \frac{\partial v_{X}}{\partial x}=0 \\
\frac{\partial v_{X}}{\partial t}-\frac{\partial v_{t}}{\partial x}=0
\end{gathered}
$$

where $u_{x}=\partial u / \partial x$ is the axial strain, $u_{t}=\partial u / \partial t$ is the axial velocity, $v_{x}=\partial v / \partial x$ is the rotational strain and $v_{t}=\partial v / \partial t$ is the rotational velocity. 


\section{FINITE ELEMENT METHOD}

For the present work, the finite element method, based on the Galerkine variational formulation, was used. Explanations of the general procedure can be found in such texts as Zienkiewicz and Taylor [23], Norrie and de Vries [20] and Buchanan [10].

The spring is divided into $n$ equal elements with two nodes and four degrees of freedom per node $\left(u_{t}, u_{X}, v_{t}, v_{X}\right)$.

For each node $i$, the adopted shape functions $N_{i}^{(j)}(x)$ are the linear ones of the roof form is plotted in Fig. 2. These functions are defined by:

$$
N_{i}^{(j)}(x)=\left\{\begin{array}{lll}
\frac{x-x_{i-1}}{x_{i}-x_{i-1}} & \text { if } & j=i-1 \\
\frac{x-x_{i+1}}{x_{i+1}-x_{i}} & \text { if } & j=i \\
0 & \text { if } & j \neq i-1 \text { and } j \neq i
\end{array}\right.
$$

$x_{i}$ and $x_{i+1}$ indicates the node abscissa bounding the element (i).

As it can be seen, $N_{i}^{(j)}=0$ for all elements $(j)$ not bounded by node $i$.

The variables $u_{t}, u_{X}, v_{t}$ and $v_{X}$ and the non constant coefficients $a, b$ and $c$ are assumed to be linearly variable with $x$ in each element $i$, e.g.:

$$
u_{t}=N_{i}^{(i)} u_{t_{i}}+N_{i+1}^{(i)} u_{t_{i+1}} \quad a=N_{i}^{(i)} a_{i}+N_{i+1}^{(i)} a_{i+1}
$$

The Galerkin procedure, as applied to equations (9) to (12) using finite elements, requires that (Dhatt and Touzot [5]):

$$
\begin{gathered}
\sum_{j} \int_{L_{j}} N_{i}^{(j)}\left[\frac{\partial u_{t}}{\partial t}-\frac{a}{e} \frac{\partial u_{X}}{\partial x}-\frac{b}{e} \frac{\partial v_{X}}{\partial x}\right] d x=0 \\
\sum_{j} \int_{L_{j}} N_{i}^{(j)}\left[\frac{\partial u_{X}}{\partial t}-\frac{\partial u_{t}}{\partial x}\right] d x=0 \\
\sum_{j} \int_{L_{j}} N_{i}^{(j)}\left[\frac{\partial v_{t}}{\partial t}-\frac{b}{e} \frac{\partial u_{X}}{\partial x}-\frac{c}{e} \frac{\partial v_{X}}{\partial x}\right] d x=0 \\
\sum_{j} \int_{L_{j}} N_{i}^{(j)}\left[\frac{\partial v_{X}}{\partial t}-\frac{\partial v_{t}}{\partial x}\right] d x=0
\end{gathered}
$$

in which $L_{j}=\Delta x$ is the length of the spring element $(j)$.

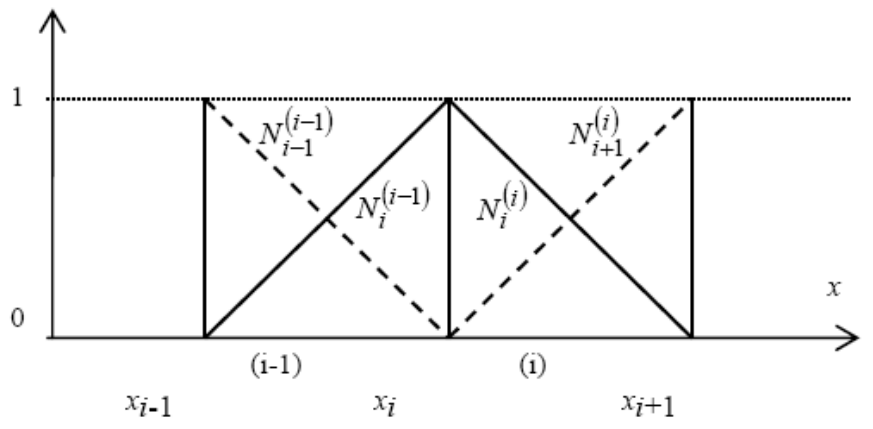

Figure 2: Functions of linear interpolation. 
Note that the sums of integrals in equations (15) to (18) are equivalently given as:

$$
\alpha \int_{x_{i-1}}^{x_{i}} N_{i}^{(i-1)}[\quad] d x+\beta \int_{x_{i}}^{x_{i+1}} N_{i}^{(i)}[\quad] d x=0
$$

in which $\alpha=\beta=1$ for all interior nodes, $\alpha=0$ and $\beta=1$ for the first node $i=1$ and $\alpha=1$ and $\beta=0$ for $i=n+1$, the last node point.

If eq. (14) and the similar equations for the other linear functions together with the linear coefficients are all substituted into equations (15) to (18) then upon using equation (19) and carrying out the indicated differentiations, there results for interior node points:

$$
\begin{gathered}
a_{i-1 / 3}\left(u_{x_{i-1}}-u_{x_{i}}\right)+b_{i-1 / 3}\left(v_{x_{i-1}}-v_{x_{i}}\right)-e \Delta x\left(\frac{\partial u_{t_{i-1}}}{\partial t}-2 \frac{\partial u_{t_{i}}}{\partial t}\right)+a_{i+1 / 3}\left(u_{x_{i}}-u_{x_{i+1}}\right) \\
+b_{i+1 / 3}\left(v_{x_{i}}-v_{x_{i+1}}\right)-e \Delta x\left(\frac{\partial u_{t_{i}}}{\partial t}-2 \frac{\partial u_{t_{i+1}}}{\partial t}\right)=0 \\
\frac{\Delta x}{6} \frac{\partial u_{x_{i-1}}}{\partial t}+\frac{2 \Delta x}{3} \frac{\partial u_{x_{i}}}{\partial t}+\frac{\Delta x}{6} \frac{\partial u_{x_{i+1}}}{\partial t}-\frac{1}{2}\left(u_{t_{i+1}}-u_{t_{i-1}}\right)=0 \\
b_{i-1 / 3}\left(u_{x_{i-1}}-u_{x_{i}}\right)+c_{i-1 / 3}\left(v_{x_{i-1}}-v_{x_{i}}\right)-e \Delta x\left(\frac{\partial v_{t_{i-1}}}{\partial t}-2 \frac{\partial v_{t_{i}}}{\partial t}\right)+b_{i+1 / 3}\left(u_{x_{i}}-u_{x_{i+1}}\right) \\
+c_{i+1 / 3}\left(v_{x_{i}}-v_{x_{i+1}}\right)-e \Delta x\left(\frac{\partial v_{t_{i}}}{\partial t}-2 \frac{\partial v_{t_{i+1}}}{\partial t}\right)=0 \\
\frac{\Delta x}{6} \frac{\partial v_{x_{i-1}}}{\partial t}+\frac{2 \Delta x}{3} \frac{\partial v_{x_{i}}}{\partial t}+\frac{\Delta x}{6} \frac{\partial v_{x_{i+1}}}{\partial t}-\frac{1}{2}\left(v_{t_{i+1}}-v_{t_{i-1}}\right)=0
\end{gathered}
$$

in which $a_{i \pm 1 / 3}=2 a_{i}+a_{i \pm 1}, b_{i \pm 1 / 3}=2 b_{i}+b_{i \pm 1}, c_{i \pm 1 / 3}=2 c_{i}+c_{i \pm 1}$, and $\Delta x=x_{i}-x_{i-1}$ is the space step size.

Since $a_{i}, b_{i}$ and $c_{i}$ are functions of $u_{x_{i}}$ and $v_{x_{i}}$, equations (20) to (23) form a coupled set of non-linear ordinary differential equations in time. In the linear case $a_{i}=a, b_{i}=b$ and $c_{i}=c$ are constants and $a_{i \pm 1 / 3}=3 a, b_{i \pm 1 / 3}=3 b$ and $c_{i \pm 1 / 3}=3 c$.

To complete the development, it is necessary to repeat the preceding steps for the boundary equations.

Equations (20) to (23) combined with (3), (4) and (5) form an equation system with $u_{t}, u_{x}$, $v_{t}$ and $v_{x}$ as the unknowns. The final step in setting up equations (20) to (23) for solution is the formulation of the scheme for integration over time. A flexible and relatively simple technique involves approximate integration over a set of finite time intervals. The approximation results from assuming that the variation in time for variables not in a time derivative can be accounted for by writing the variables as weighted averages over time, e.g.:

$$
\bar{u}_{t_{i}}=u_{t_{i}}^{k-1}+\eta\left(u_{t_{i}}^{k}-u_{t_{i}}^{k-1}\right)
$$

in which $\bar{u}_{t_{i}}$ is the weighted average between time levels $k-1$ and $k$; and $\eta$ is a parameter which affects the stability of the solution; $0 \leq \eta \leq 1$. As an example, the first term in eq. (20) would be written $\bar{a}_{i-(1 / 3)}\left(\bar{u}_{x_{i}}-\bar{u}_{x_{i-1}}\right)=\left(2 \bar{a}_{i}+\bar{a}_{i-1}\right)\left(\bar{u}_{x_{i}}-\bar{u}_{x_{i-1}}\right)$. Semi implicit Euler method is used to integrate the time derivatives over the time step $(k-1+\eta) \Delta t$ to yield terms of the form: 


$$
\frac{1}{\Delta t} \int_{(k-1) \Delta t}^{k \Delta t} \frac{\partial u_{t_{i}}}{\partial t} d t=\frac{u_{t_{i}}^{k}-u_{t_{i}}^{k-1}}{\Delta t}=\frac{\bar{u}_{t_{i}}-u_{t_{i}}^{k-1}}{\eta \Delta t}
$$

In this expression, eq. (24) was used to replace $u_{t_{i}}^{k}$.

From the characteristic theory, the strain and velocity waves propagate in the spring with speeds given by (Ayadi et al. [4]):

$$
c_{S}=\left[\frac{(a+c)+\sqrt{(a-c)^{2}+4 b^{2}}}{2\left(a c-b^{2}\right)}\right]^{-1 / 2} \text { and } c_{f}=\left[\frac{(a+c)-\sqrt{(a-c)^{2}+4 b^{2}}}{2\left(a c-b^{2}\right)}\right]^{-1 / 2}
$$

$c_{f}$ is the fast speed of rotational waves $\left(v_{x}, v_{t}\right)$ and $c_{s}$ is the slow speed of axial waves $\left(u_{x}, u_{t}\right)$.

When linear theory is used, we obtain:

$$
c_{f}=\sqrt{\frac{\sin \alpha}{e}} \text { and } c_{s}=\sqrt{\frac{\sin \alpha}{e(1+v)}}
$$

These speeds are used to define the time step $\Delta t=t^{k}-t^{k-1}$ from the Courant-FreidrichsLewy [22] stability condition for finite difference approximations of hyperbolic equations:

$$
c_{f} \frac{\Delta t}{\Delta x} \leq 1
$$

Time integration of equations (20) to (23), which involves the use of equations (24) and (25), yields a set of non-linear equations that are frequently solved by using iterative algorithms. After assembly, we obtain the non-linear matrix system:

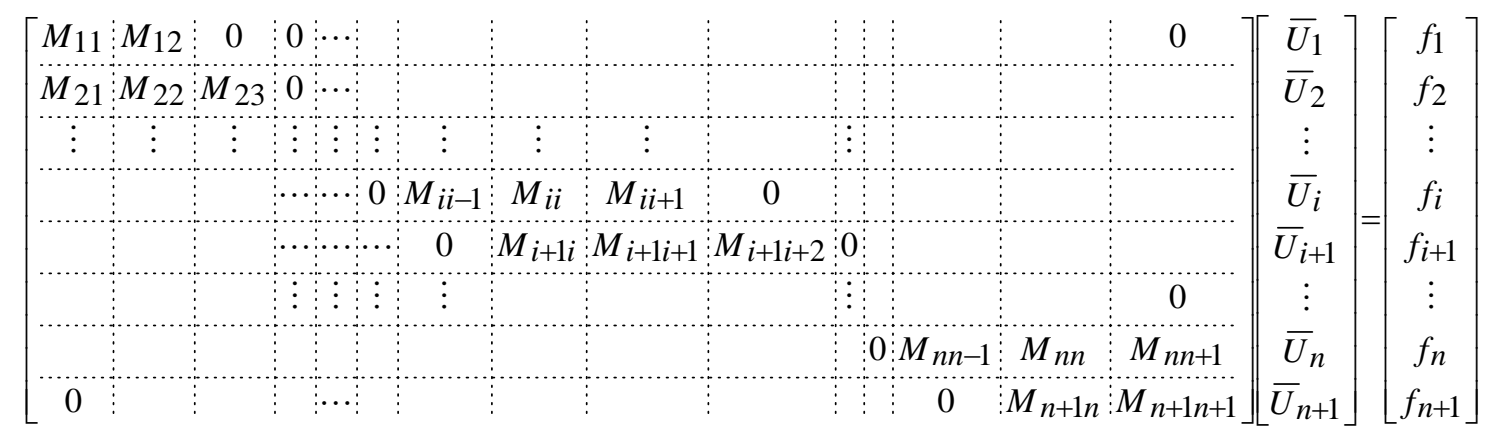

where:

$-M_{i k}$ is (4x4) matrix which is null if $k<i-1$ or $k>i+1$ else it depends of the unknowns of the problem $\left\{\bar{u}_{x_{i-1}}, \bar{v}_{x_{i-1}} \quad \bar{u}_{x_{i}}, \bar{v}_{x_{i}} \quad \bar{u}_{x_{i+1}}, \bar{v}_{x_{i+1}}\right\}$ at the time $(k-1+\eta) \Delta t$ and $(k-1) \Delta t$.

- $\left\{\bar{U}_{i}\right\}^{t}=\left(\bar{u}_{t_{i}}, \bar{u}_{x_{i}}, \bar{v}_{t_{i}}, \bar{v}_{x_{i}}\right)$ is the unknown vector at the node $i$ and the time $(k-1+\eta) \Delta t$.

- $f_{i}$ is a second member vector which depends of the unknowns of the problem at the time $(k-1) \Delta t$.

- $M_{11}, M_{12}, M_{n+1 n}, M_{n+1 n+1}$ are (4x4) matrix which depends of the boundary conditions in the nodes 1 and $n+1$.

- $f_{1}$ and $f_{n+1}$ contain the boundary conditions.

The final step in the procedure is the solution of the non-linear matrix system (29) written as a compact form:

$$
[K(\bar{U})]\{\bar{U}\}=\{f\}
$$


where $\bar{U}$ is the solution at the time $(k-1+\eta) \Delta t$.

The solution of the system (30) is obtained using an iterative algorithm based on the Gauss substitution method (Scheid [19]). In this method a succession of solutions $\left\{\bar{U}^{1}\right\},\left\{\bar{U}^{2}\right\}, \ldots\left\{\bar{U}^{m}\right\}$ are constructed. $\left\{\bar{U}^{m}\right\}$ is calculated from $\left\{\bar{U}^{m-1}\right\}$ by solving the following linear system:

$$
\left\lfloor K\left(\{\bar{U}\}^{m-1}\right)\right\rfloor\{\bar{U}\}^{m}=\{f\}, \quad m=1,2, \cdots
$$

with $\{\bar{U}\}^{0}=\{U\}^{k-1}\left(\{U\}^{k-1}\right.$ is the solution at the time $\left.(k-1) \Delta t\right)$.

The process is repeated until the convergence of solution. This is obtained when $\left\|\{\bar{U}\}^{m}-\{\bar{U}\}^{m-1}\right\| \leq \varepsilon\left\|\{\bar{U}\}^{m-1}\right\|$, where $\varepsilon<<1$.

The stability and the speed of convergence depend of three parameters which are the time increment $\Delta t$, the number of finite element $n$ and the value of $\eta$ which must satisfy the relation (28) and $\eta \geq 0.5$ (Richtmeyer and Morton [24]).

When the solution $\{\bar{U}\}$ is obtained at the time $(k-1+\eta) \Delta t$, eq. (24) permits us to obtain the solution $\{U\}^{k}$ at the time $k \Delta t$.

\section{NUMERICAL RESULTS FOR IMPACTED SPRING}

Consider the hypothetical spring system shown by Fig. 1.b. The parameters of the spring are:

- the original length of spring $h=48.26 \mathrm{~cm}$,

- the helix angle $\alpha=0.141815 \mathrm{rd}$,

- the radius of the spring $r=17.32 \mathrm{~cm}$,

- the number of coils $n=3$,

- the Poisson's ratio $v=0.29$,

- the wire radius $r_{f}=1.509 \mathrm{~cm}$,

- the Young's modulus $E=20.685 \cdot 10^{6} \mathrm{~N} / \mathrm{cm}^{2}$,

- the initial compression $\Delta=16.51 \mathrm{~cm}$ and the mass of the spring $M=19.146 \mathrm{~kg}$.

\subsection{Initial conditions}

The initial conditions are (Fig. 1.b):

$$
\begin{array}{cc}
u_{x}(x, 0)=-\Delta / h & v_{x}(x, 0)=0 \\
u_{t}(x, 0)=0 & v_{t}(x, 0)=0
\end{array}
$$

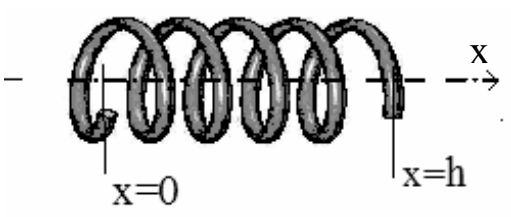

Figure 3: Helical spring boundaries.

\subsection{Boundary conditions}

The dynamic response studied here is due to a given velocity $\phi_{1}(t)$ at the impacted end of the spring $x=0$ (Fig. 3). This velocity is used by Sinha and Costello [13] and is shown in Fig. 4. 
The boundary conditions are:

$$
u_{t}(0, t)=\phi_{1}(t), v_{t}(0, t)=0, u_{t}(h, t)=0 \text { and } v_{t}(h, t)=0
$$

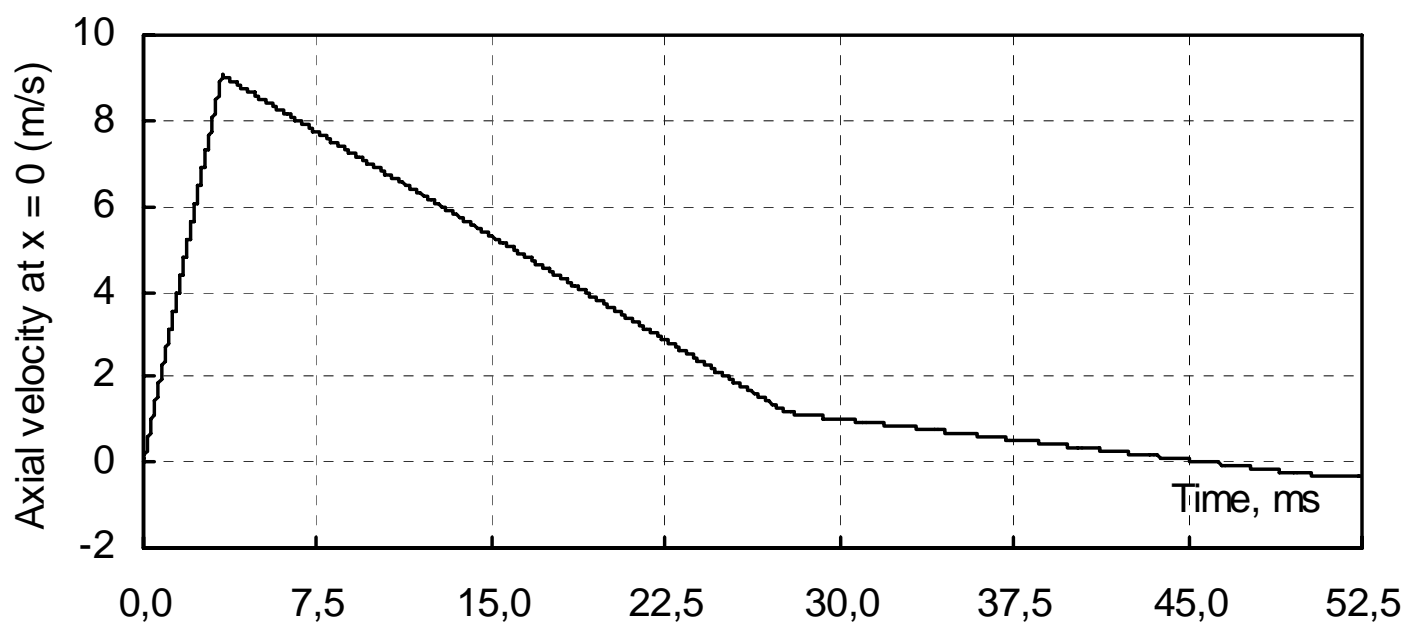

Figure 4: Axial velocity at the impacted end of the spring $x=0$.

For the numerical resolution, a general-purpose computer program, based on the finite element method (FEM), is coded in FORTRAN to analyse the dynamic response of helical spring.

The spring is divided into $n$ elements in the $x$ direction: $\Delta x=h / n$. For the present work, the figures illustrate the results obtained by the FEM with a number of elements $n=100$ and $\eta=0.6$.

\subsection{The wavespeeds}

Fig. 5 represents the evolution of the wavespeeds, defined by equations (26) and (27), at some sections of the spring. When linear theory is used, the wavespeeds are constant. But, when the non-linear theory is applied, due to the non-linearity of equations (1) and (2), the wavespeeds are not constant. As it can be seen from Fig. 5, the fast rotational strain wave propagates with a wavespeed varying in the range $30.731 \leq c_{f} \leq 31.171 \mathrm{~m} / \mathrm{s}$ and is always bigger than the linear wavespeed $c_{f}=30.55 \mathrm{~m} / \mathrm{s}$. While, the slow axial strain wave propagates with a wavespeed varying in the range $25.623 \leq c_{s} \leq 26.547 \mathrm{~m} / \mathrm{s}$ and is always smaller than the linear value $c_{s}=$ $26.898 \mathrm{~m} / \mathrm{s}$.

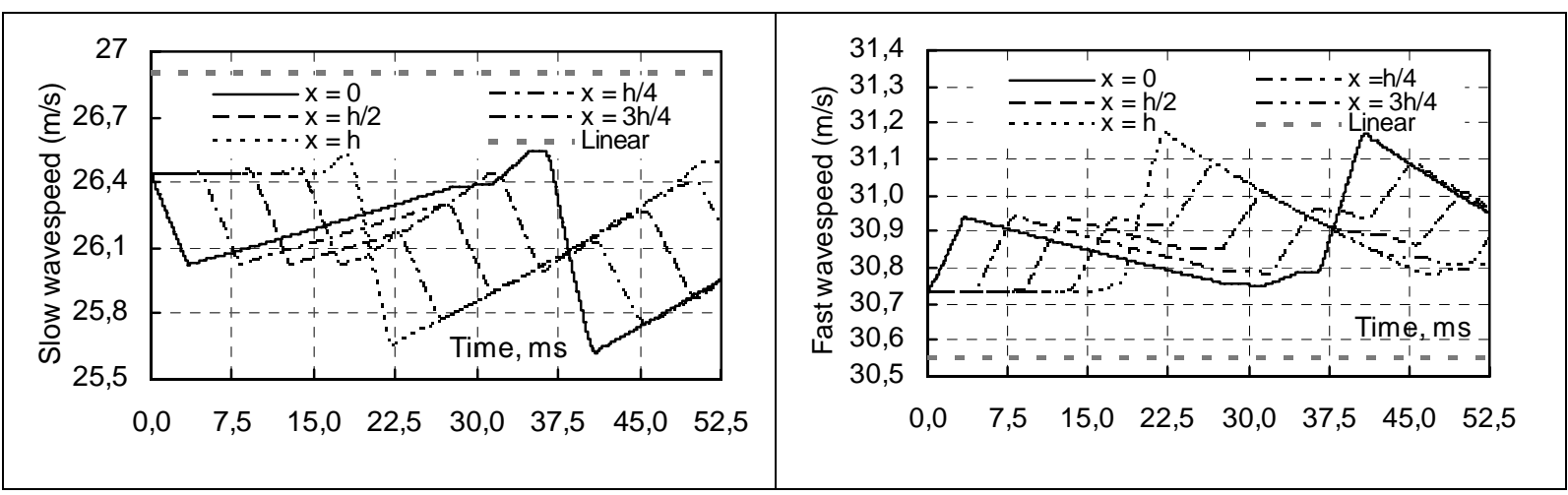

Figure 5: Slow and fast wavespeeds in the spring. 


\subsection{Numerical validation}

To validate the concept of modelling retained herein, the evolution of axial and rotational strains at the end $x=0$, following the loading imposed at this end, is presented in the Fig. 6 . The results from the FEM show a good agreement with the numerical solution of Sinha and Costello [13] obtained by the method of characteristics.

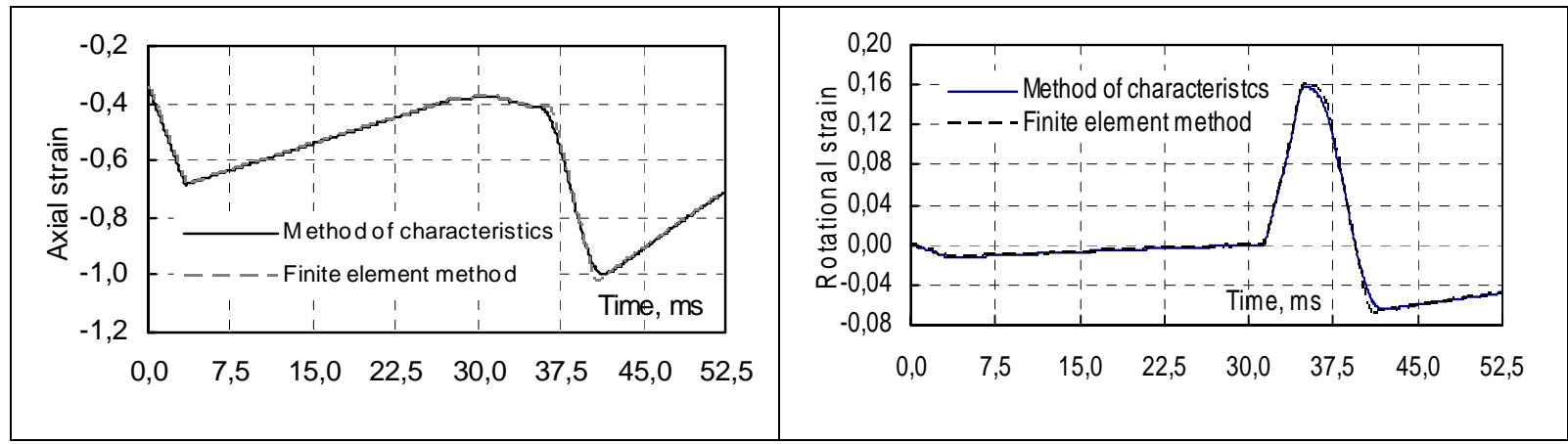

Figure 6: Comparison between the finite element method and the method of characteristics [13].

\subsection{Physical interpretation of the wave propagation in the spring}

Figs. 7 and 8 show the phenomena of linear and non-linear dynamic responses of the helical spring. The computed axial and rotational strains are plotted at some sections of the spring ( $x=0, x=h / 4, x=h / 2, x=3 \cdot h / 4$ and $x=h$ ). These results where obtained from linear and non-linear models using the finite element method. The evolution of strains would result from the velocity function applied at the impacted end, $x=0$, and from the wave reflections at the two ends of the spring.

It can be noted from these figures that, at time $t=0$, the end of the spring $x=0$ is excited. Immediately, two waves of strains, one rotational $\beta$ and the other axial $\varepsilon$ propagate towards the end $x=h$ with two different speeds. The wave amplitudes depend on the excitation and geometrical and mechanical properties of the spring.

The axial and rotational strain waves, travelling along the spring, show amplification of these strains at the two ends of the spring generally attributed to the effects of reflections. That is, the reflected waves are more amplified than the incident ones. Moreover, the slow axial strain wave has a very clear influence on the rotational strain. In fact, the reflected fast rotational strain wave at the fixed end of the spring $(x=h)$ causes rotational strain to rise. But, the reflected axial wave attenuates and limits the values of the rotational strain. The results indicate the influence of the axial strain wave on the behaviour of the rotational strain in the spring for the applied loading. This influence represents the Poisson coupling relating the axial to the rotational waves through the radial contraction or expansion of the spring. It is named after Poisson in connection with the contraction coefficient $v$. Poisson coupling leads to precursor waves. These are rotational-wave induced disturbances in the spring which travel faster and hence in front of, the axial waves. Due to this interaction mechanism, there is also an influence of the rotational wave on the axial strain, but it is not well demonstrated in figures because of scales.

Fig. 7 shows that there is some discrepancy between the linear and non-linear axial strain-time curves. As the linear speed is bigger than the non-linear one, the changes in the amplitude of the axial linear waves are produced before the changes of the non-linear ones. In opposite, for the rotational strain, as it can be seen in Fig. 8, the changes of the linear wave amplitudes are produced after the non-linear ones. 

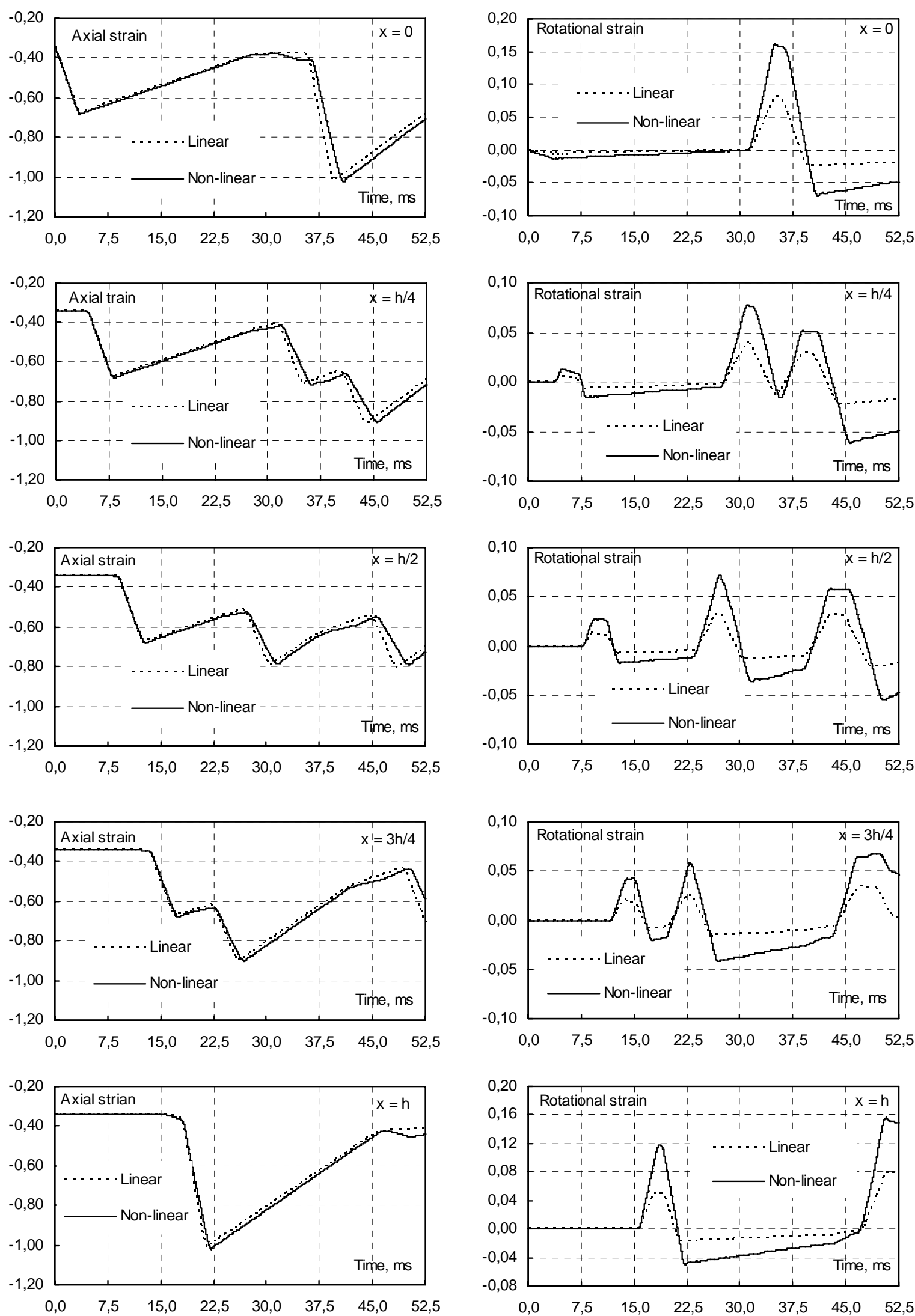

Figure 7: Axial strains in the spring.

Figure 8: Rotational strains in the spring.

Fig. 9 shows the evolution of axial and rotational velocities at the same sections of the spring $(x=0, x=h / 4, x=h / 2, x=3 \cdot h / 4$ and $x=h)$ and for the considered loading. 
The dynamic behaviour of the axial and rotational velocities indicates that the maximal positive velocity is the same in all sections of the spring. It is equal to the maximal value of the excitation at $x=0$. But the minimal negative velocities are not the same for all sections of the spring.

The same conclusions may be drawn for the comparison between the linear and non-linear velocity waves in the spring.

In general, it can be noted that the values obtained for the rotational waves by the non-linear model are more important than those issued from the linear one.

As pointed by Phillips and Costello [12], the results of the plots show the necessity of solving the non-linear equations of motion for the spring under this type of loading. The linear theory is adequate for predicting the axial force in the spring but can lead to erroneous results in predicting the axial twisting moment and radial expansion of the spring.

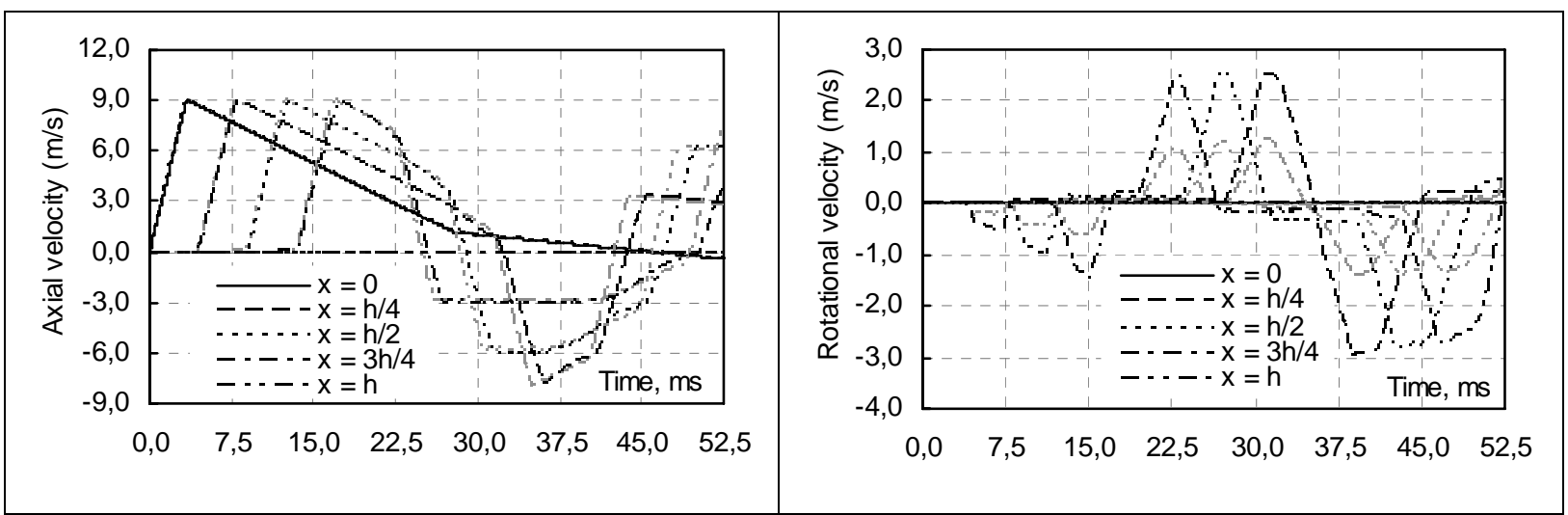

Figure 9: Axial and rotational velocities in the spring.

Non-linear

Linear

\section{CONCLUSION}

The strain and velocity waves propagation in cylindrical helical springs made of elastic materials is investigated in this study. This problem is governed by two coupled non-linear partial differential equations of the hyperbolic type. When the strains are supposed small, the coefficients are constant and the equations of motion are rendered linear. The numerical finite element method, using the Galerkin technique, is employed to solve both linear and non-linear formulations. This method transforms the partial derivative equations into a non-linear system of equations solved by an iterative algorithm, based on the Gauss substitution method. The obtained results permit to analyze the evolution of axial and rotational strains and velocities in various sections of the spring due to an axial loading. Poisson-coupling induced rotational waves were predicted and observed. This coupling process indicates the influence of axial strain wave on the behaviour of rotational strain. To validate the reliability of the model presented, the numerical results were confronted successfully with the numerical solution of the studied problem obtained by the method of characteristics. The curves of the strains and velocities, in some sections of the spring, show that the amplitudes of axial and rotational waves depend on the excitation and the geometrical and mechanical characteristics of the spring. It can be seen from the calculated results that the linear theory is reasonably accurate as far as the axial strain is concerned but is in considerable error for investigating the rotational strain.

The framework developed in this paper can be continued to study the influence of the friction force on the behaviour of the strains and velocities in the spring. 


\section{REFERENCES}

[1] Love, E. H. (1927). A Treatise on the Mathematical Theory of Elasticity, 4th Editions Dover, New York, 415

[2] Ayadi, S.; Hadj-Taïeb, E. (2006). Simulation numérique du comportement dynamique linéaire des ressorts hélicoïdaux, Transactions the Canadian Society of Mechanical Engineering, Vol. 30, No. 2, 191-208

[3] Ayadi, S.; Hadj-Taïeb, E. (2006). Influence des caractéristiques mécaniques sur la propagation des ondes de déformations linéaires dans les ressorts, Mécanique \& Industrie, Vol. 7, No. 5-6, 551-563

[4] Ayadi, S.; Hadj-Taïeb, E.; Pluvinage, G. (2007). The numerical solution of strain waves in elastical helical spring, Materiali in Technologijie, Vol. 41, No. 1, 47-52

[5] Dhatt, G.; Touzot, G. (1984). Une présentation de la méthode des éléments finis, Edition Maloine SA, Paris

[6] Cook, R. D.; Young, W. C. (1985). Advanced Mechanics of Materials, New York, Macmillan Publishing Company

[7] Costello, G. A. (1975). Radial expansion of impacted helical springs, Journal of Applied Mechanics, Transaction. ASME, Vol. 42, 789-792

[8] Damak, F.; Taktak, M.; Abid, S.; Dhieb, A.; Haddar, M. (2005). Finite element method for the stress analysis of isotropic cylindrical helical spring, European Journal of Mechanics A/Solids 24, Vol. 12, 1068-1078

[9] Forrester, M. K. (2001). Stiffness model of a die spring, Thesis of Master Science doctorate, Faculty of the Virginia Polytechnic Institute

[10] Buchanan, G. R. (2004). Finite Element Analysis, Tata McGraw-Hill Edition, New Delhi, 143

[11] Mottershead, J. E. (1982). The large displacements and dynamic stability of spring using helical finite elements, International Journal of Mechanical Sciences, Vol. 24, 547-558

[12] Phillips, J. W.; Costello, G. A. (1972). Large deflections of impacted helical springs, The Journal of the Acoustical Society of America, Vol. 51, 967-972

[13] Sinha, S. K.; Costello, G. A. (1978). The numerical solution of the dynamic response of helical springs, International Journal for Numerical Methods in Engineering, Vol. 12, 949-961

[14] Stokes, V. K (1974). On the dynamic radial expansion of helical springs due to longitudinal impact, Journal of Sound and Vibration, Vol. 35, 77

[15] Yildirim, V.; Ince, N. (1997). Natural frequencies of helical springs of arbitrary shape, Journal of Sound and Vibration, Vol. 204, No. 2, 311-329

[16] Yildirim, V. (1998). An efficient numercal method for predicting the natural frequencies of cylindricial helical springs, International Journal of Mechanical Sciences, Vol. 41, 919-939

[17] Yildirim, V. (2002). Expressions for predicting fundamental natural frequencies of non-cylindrical helical springs, Journal of Sound and Vibration, Vol. 252, No. 3, 479-491

[18] Becker, L. E.; Chassie, G. G.; Cleghorn, W. L. (2002). On the natural frequencies of helical compression springs, International Journal of Mechanical Sciences, Vol. 44, 825-841

[19] Scheid, F. (2004). Numerical Analysis, Second Edition, Tata McGraw-Hill, New Delhi, 354

[20] Norrie, D. H.; Vries, G. (1973). The Finite Element Method, Academy Press Inc., New York

[21] Desai, C. S.; Abel, J. F. (1972). Introduction to the Finite Element Method, Van Nostrand Reinhold Co, New York

[22] Courant, F. R.; Lewy, K. H. (1928). Uber die Partiellen Differenzengleichungen der Mathematischen Physick, Math. Annaen, Vol. 100, 32-74

[23] Zienkiewicz, C.; Taylor, R. L. (1988). The Finite Element Method, The fourth edition, McGraw-Hill Book Company, U.K.

[24] Richtmeyer, R. D.; Morton, K. W. (1967). Difference methods for initial value problems, Intersciences Publishers, A Division of John Wiley and Sons, New York

[25] Yutaka, T.; Jong-Bin, L.; Minoru, T. (2004). Finite element analysis of superelastic, large deformation behavior of shape memory alloy helical springs, Computers and Structures, Vol. 82, $1685-1693$

[26] Lee, J.; Thomson, D. J. (2000). Dynamic stiffness formulation, free vibration and wave motion of helicalm springs, Journal of Sound and Vibration, Vol. 239, No. 2, 297-320 